\section{The effect of memory load on reaction time in character classification*}

\author{
BLAKE L. WATTENBARGER \\ Bell Telephone Laboratories, Holmdel, New Jersey 07733 \\ and \\ ROBERT G. PACHELLA $†$ \\ The University of Michigan, Ann Arbor, Michigan 48104
}

In order to investigate the effect of memory load on reaction time (RT), choice RT trials were embedded in a binary character classification task using the varied set procedure. Twelve Ss performed in experimental blocks, as well as in control blocks consisting of character classification trials only. In experimental blocks, every trial began as a classification trial with the presentation of a new positive set. On a random half of these trials, however, a choice RT stimulus was presented instead of a probe letter and S simply made the indicated response. Results indicated that memory load had no effect on the choice RT trials. Embedding choice RT trials in the classification task affected the intercept (but not the slope) of the function relating classification RT to memory load. This result implies that the increase in latency usually obtained in classification experiments is entirely due to an increase in the duration of the memory searching stage of processing.

Recent interest in the processes underlying character classification has placed an increased emphasis on the relationship between the amount of information stored in short-term memory and the reaction times (RT) obtained in information-processing tasks. In a typical paradigm (Sternberg, 1969), a $S$ is required to memorize a short list of characters (usually letters or digits) before each trial. A single probe character is then presented, and the $S$ is required to decide whether or not this character is contained in the memorized list. RT, measured from the onset of the probe character, is typically found to be an increasing function of the size of the memorized list.

One long-standing conception of man as a single-channel information processor would hold that such a task involves a division of the total limited processing capacity available to the $S$ (Moray, 1967). That is, in such a task, the $S$ is required to maintain items in memory at the same time that he is attempting to encode the probe character. It is possible, according to this view, that the amount of capacity available for the encoding function

*This research was supported by the Advanced Research Projects Agency, U.S. Department of Defense, and monitored by the U.S. Air Force Office of Scientific Research, under contracts AF 49(638)-1736 and F44620-72-C-0019 with the University of Michigan's Human Performance Center, Department of Psychology. The authors would like to thank Roberta L. Klatzky for her constructive criticism of an earlier version of the present paper.

tAddress: Human Performance Center, 330 Packard Road, Ann Arbor, Michigan 48104. could, to some extent, be limited by the amount of capacity needed to maintain information in short-term memory. If increasing the amount of information in memory increases the amount of capacity needed for that function, then some increase in RT might be expected due to the depleted capacity available for the encoding function. This is not to imply that all of the increase in RT found in character classification studies is attributable to a division of processing capacity between the memorial and the encoding aspects of that task, but rather that the division of processing capacity may lead to some increase in the duration of the encoding stage of processing.

Several experiments provide support for this interpretation. In a study by Posner and Rossman (1965), Ss were required to hold three-digit numbers in memory while performing various information reduction tasks. The rate of forgetting increased when more information reduction was required in the intervening task. In a similar study by Crowder (1967), the Ss held five unrelated nouns in memory while performing a serial RT task. Compatibility of the RT task was varied, and the low-compatibility condition produced more forgetting than the high-compatibility condition. Furthermore, Murdock (1965) has shown not only that increasing the information content of an interpolated information-processing task increasingly depresses recall of a memorized list, but also that Ss are capable of trading off the relative performances of the two tasks.

In an experiment somewhat more relevant to the considerations raised above, Sanders and van Borselen (1966) measured choice RT for Ss who were simultaneously engaged in a continuing memory span task for single digits. They found that when Ss had a memory bias, choice RT increased systematically for memory loads of 0,1 , or 2 digits in memory. Likewise, Shulman and Greenberg (1971) have noted increases in choice RT when lists of 6 or 8 consonants were being held in memory simultaneously. List Length 4, however, did not produce RTs different from lists of 0 and 2 consonants. Thus, it is possible that only memory loads which exceed memory span length will have systematic effects on RT. It should be noted in this connection that the continuing memory span task of Sanders and van Borselen (1966) also considerably tested S's memory capacity. 1 on the other hand, Sternberg (1967) found that, at least early in practice, increasing memory loads systematically increased the effect of stimulus degradation on RT in a character classification task involving memorized lists of 1,2 , or 4 digits. Sternberg interpreted this finding as evidence that stimulus degradation had an effect on the memory scanning processes of character classification, but it is alternatively possible that memory load might, in fact, systematically affect the encoding stages of this task. The present experiment represents a converging operation that should remove this particular ambiguity. It is, furthermore, hoped that the present design will be more sensitive than either the Shulman and Greenberg (1971) or the Sanders and van Borselen (1966) experiments in showing any subtle effect on RT of relatively small memory loads like those encountered in character classification.

\section{METHOD}

Subjects

The Ss were 12 right-handed students at the University of Michigan, 6 men and 6 women. They volunteered to serve in the experiment and were paid for their services.

\section{Apparatus}

Stimuli and feedback were presented on a DEC Type 30 cathode ray tube display, controlled by a PDP-1 computer. The $S$ sat facing the display in a small, dimly lighted room; the distance between the screen and S's eyes was approximately $70 \mathrm{~cm}$ (28 in.). Immediately below the screen on a sloping surface in easy reach for the $S$, there was a response keyboard with two keys. The keys required a minimum force of about $0.5 \mathrm{~N}(51 \mathrm{~g})$ 
for activation. Low-level white noise was presented to the $S$ through earphones to mask distracting noises. The $E$ could interrupt the noise and speak to the $S$ through the earphones, and the $S$ could answer through a nearby intercom that was kept on continuously.

\section{Experimental Design}

The character classification task was used, with only minor modifications, in the experimental condition. Each trial began with presentation of a new positive set of one to five uppercase letters. The probe could be either an uppercase letter or an arrow. If the probe was a letter, the $\mathbf{S}$ performed the usual classification task, indicating with a keypress whether or not the letter was in the positive set; the right key was used for the positive response and the left key for the negative response. If the probe was an arrow, it pointed at an angle either down to the left or down to the right. The $S$ was instructed simply to press the key toward which the arrow pointed. Half of the trials in the experimental condition were of each type; the former will be referred to as embedded classification trials, and the latter as choice RT trials. Thus, every trial began as a classification trial, but classification was required only half of the time; trials of the two types were randomly intermixed, so the $S$ could not anticipate a trial of either type. In the control condition, only classification trials were presented.

Each $\mathbf{S}$ served for about $\mathbf{3} \mathrm{h}$, divided into two sessions. Each session was divided into seven blocks of 44 trials. The first block of a session and the first 4 trials of each subsequent block were for practice and warm-up, and the data from those trials were discarded. The control condition was used for the practice block at the beginning of the session.

Two punched paper tapes were prepared to control the presentation of stimuli, each tape containing the seven blocks of one session. After the initial practice block, the blocks alternated between experimental $(E)$ and control (C) conditions, but in opposite orders for the two tapes. Thus, the order for the seven blocks of Tape 1 was CECECEC, and the order for Tape 2 was CCECECE. Each S received both tapes; half received Tape 1 followed by Tape 2, and half received them in the reverse order. In each session, the Ss were given $5 \cdot \mathrm{min}$ breaks after the third and fifth blocks, and brief $(30$-sec) breaks after the other blocks.

Excluding the warm-up trials, each block in the control condition included eight trials with each set size from one to five. The positive response was correct on half of the trials with each set size; the negative response was correct on the remaining trials. Thus, there were four trials with each response for each set size; the resulting 40 trials were presented in a random order. The experimental condition blocks were prepared by first constructing the block as if it were for the control condition, then replacing the probe letter with an arrow on half of the trials with each set size and response. Right-pointing arrows were inserted on trials that would have required a positive (right) response, and vice versa. Thus, there were equal numbers of right- and left-response choice RT trials for each set size in each block. For both experimental and control blocks, on positive classification trials, the serial position of the letter in the positive set that matched the probe was balanced within blocks as nearly as possible.

The letters used as stimuli were 10 consonants (B, D, G, H, J, M, N, Q, R, and $T$ ). Each positive set of Size $s$ was formed by taking the $s$ letters in a random permutation of the 10 consonants. These letters were carefully programmed for the display to resemble Metro Thin No.2 typeface, which was chosen for its simplicity and normative appearance. The letters, as they appeared on the display screen, were $2.4 \mathrm{~cm}$ (0.95 in.) high.

A description of the task was read to the $S$ at the beginning of the first session. The Ss were told before each block wehther there would be "arrow trials" in that block. They were instructed to respond as quickly as possible, but more emphasis was put on accuracy than speed.

Each trial began with sequential presentation of the positive set. The first letter was presented in the upper left part of the screen, the second to the right of the first, etc. Each letter appeared in a rectangle $3.1 \mathrm{~cm}$ high and $2.2 \mathrm{~cm}$ wide (1.2 $\times 0.87$ in.), remained on for $1.2 \mathrm{sec}$, and was then masked by a solid white field for 0.5 sec. The masking was made necessary by the long persistence phosphor (P7) used for this display. The next letter appeared immediately on termination of the mask of the preceding one. A delay of $2.0 \mathrm{sec}$ intervened between the termination of the mask of the last letter in the positive set and the onset of the warning signal, which was the appearance of a rectangle in the center of the screen. One second later, the probe appeared in the rectangle and the computer began timing the S's RT. The probe and rectangle disappeared when the $\mathrm{S}$ responded, and the words "correct" or "error" (whichever was appropriate) appeared in the lower left corner of the screen for $2.0 \mathrm{sec}$. Between termination of the feedback and the beginning of the next trial there was a delay of $0.5 \mathrm{sec}$.

\section{RESULTS}

Mean RT for correct responses is shown as a function of set size for each condition in Fig. 1. The data have been averaged over Ss, sessions, and responses. The straight lines shown were fitted by the least-squares method.

Straight lines were fitted to each S's data separately. Two lines, one for each response, were fitted for each condition on each session. The slopes and intercepts of these lines were submitted to separate three-way analyses of variance, with the factors of conditions, sessions, responses, and Ss. The analysis of the slopes revealed only a main effect of conditions $[F(2,22)=66.336, p<.001]$. The analysis of the intercepts revealed significant main effects of sessions $[F(1,11)=42.189, p<.001]$ and responses $[F(1,11)=8.839, p<.05]$ and a significant interaction of Conditions by Responses $[F(2,22)=$ $3.547, \mathrm{p}<.05]$. The main effect of conditions approached significance at the $\mathrm{p}=.05$ level $[\mathrm{F}(2,22)=3.117$, $\mathrm{p}=.0641$.

Inspection of Fig. 1 suggests that the main effect of conditions on the slopes was due to the small slope of the choice RT condition. In fact, the slopes of the control and embedded classification conditions were not different $[t(11)=1.860, p>.05]$. The slope of the choice RT condition was not significantly different from zero $[t(11)=1.368, p>.05] .^{2}$

Although the analysis of variance of the intercepts did not reveal a main effect of conditions, the increase in the intercept of the embedded classification over the control conditions was significant by a one-tailed $t$ test $[t(11)=1.921$, $\mathrm{p}<.05]$

The error rates for the control and embedded classification conditions were $2.85 \%$ and $2.99 \%$, respectively. The error rate for choice RT trials was $0.14 \%$.

\section{DISCUSSION}

Choice RT was unaffected by memory load over the range studied. Since the range of memory loads was selected to correspond to the range typically used in character classification tasks, the possibility can be ruled out that part of the increase in RT found for such tasks is due to increasing the duration of the encoding stages of processing.

Confidence in this conclusion requires evidence that $S s$ were, in fact, holding the memorized list in 
short-term memory on choice RT trials. That evidence is provided by the data from embedded classification trials. On those trials, the search process proceeded at the usual rate, with only a constant increase in overall RT. If Ss had not held the memory set in short-term memory on experimental trials (holding it in long-term memory instead, for example), the search rate on embedded classification trials would have been considerably slower. Sternberg (1969, Experiment 5) found the search rate nearly halved when Ss were not allowed to hold the positive set in short-term memory.

The conclusion being drawn from the present data, that memory load does not affect the encoding stage in character classification, is based on assumptions about the similarity of the processes involved in the choice RT and classification tasks. It has been assumed, for example, that the stimulus encoding processes for the two tasks must be the same. It might also be concluded that, following stimulus encoding on each kind of trial, a "task" decision is made during which the $S$ determines what kind of trial, choice RT or classification, is taking place. Such a stage can be inferred from the constant difference between the experimental and control classification conditions.

It might also be assumed that the response decision, translation, and organization processes are also the same for both tasks: in other words, that the only difference between the choice RT and classification trials is the inclusion of a memory scanning stage in the latter. This assumption can be shown to be unwarranted on the basis of the present data. The analysis of variance of the intercepts yielded a significant main effect of responses and a significant Response by Condition interaction. The main effect of responses derives from the difference of $70.5 \mathrm{msec}$ between the right-key and left-key responses on classification trials (averaged over control and embedded classification conditions); the right-key (yes) response was faster. The interaction was significant because the corresponding difference between responses for the choice $\mathrm{RT}$ condition was $1.1 \mathrm{msec}$ in the opposite direction. Clearly, there is a real difference between positive and negative responses in the classification task that

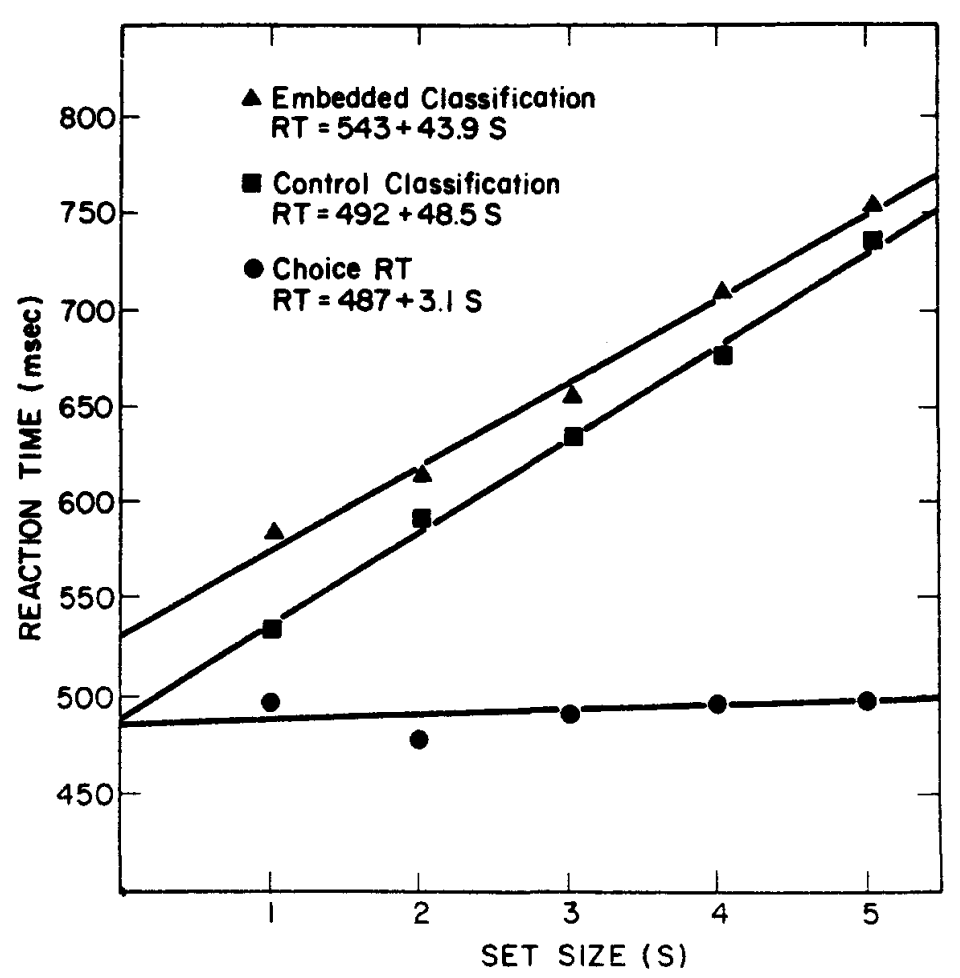

Fig. 1. Reaction time as a function of size of positive set for the experimental and control conditions.

is not attributable to handedness or other experimental artifacts, and thus, there must be differences between the choice RT and classification trials that are not simply attributable to the presence of memory scanning in the classification task.

Regardless of these assumptions, the fact that small memory load differences produce no effect on choice RT confirms the finding of Shulman and Greenberg (1971). The division of capacity between memorial and perceptual processing seems only to be important when some upper limit of total processing capacity is exceeded, and the independence of distinct processing mechanisms will be maintained so long as this bound is not reached.

\section{REFERENCES}

CROWDER, R. G. Short-term memory for words with a perceptual-motor interpolated activity. Journal of Verbal Learning \& Verbal Behavior, 1967, 6, 753-761.

MORAY, N. Where is capacity limited? A survey and model. In A. F. Sanders (Ed.) Attention and performance. Amsterdam:
North Holland, 1967.

MURDOCK, B. B. Effects of subsidiary task on short-term memory. British Journal of Psychology, 1965, 56, 413-419.

POSNER, M. I., \& ROSSMAN, E. Effect of size and location of informational transforms upon short-term retention. Journal of Experimental Psychology. J965,70, 496-505.

SANDERS, A. F., \& van BORSELEN, J. W. Continuing memory and information processing. Joumal of Experimental Psychology, 1966, 71, 844-848.

SHULMAN, H. G., \& GREENBERG, S. N Perceptual deficit due to division of attention between memory and perception. Journal of Experimental Psychology, 1971, 88, 171-176.

STERNBERG, $S$. Two operations in character recognition: Some evidence from reaction-time measurements. Perception \& Psychophysics, 1967, 2, 43-53.

STERNBERG, S. Memory-scanning: Mental processes revealed by reaction-time experiments. American Scientist, 1969 $57,421-457$.

NOTES

Sanders, personal communication, August 1971 .

2. Averaged over sessions and responses, was negative and for seven was positive.

(Accepted for publication April 20, 1972.) the slope of choice RT function for five $\mathrm{Ss}$ 